\title{
A Mathematical Model of HIV Transmission in Homosexuals With Genetic Heterogeneity
}

\author{
SHU-FANG HSU SCHMITZ*
}

Institut für Mathematische Statistik und Versicherungslehre (IMSV), Universität Bern, 3012 Bern, Switzerland

(Received 22 November 1998; In final form 9 August 1999)

\begin{abstract}
Several AIDS cohort studies observe that the incubation period between HIV infection and AIDS onset can be shorter than 3 years in about $10 \%$ seropositive individuals, or longer than 10 years in about $10-15 \%$ individuals. On the other hand, many individuals remain seronegative even after multiple exposures to HIV. These distinct outcomes have recently been correlated with some mutant genes in HIV co-receptors (e.g., CCR5,CCR2 and CXCR4). For instance, the mutant alleles $\triangle 32$ and m303 of CCR5 may provide full protection against HIV infection in homozygotes and partial protection in heterozygotes; moreover, infected heterozygotes may progress more slowly than individuals who have no mutant alleles. Frequencies of these mutant alleles are not very low in Caucasian populations, therefore, their effects may not be insignificant. Based on available data, we propose a onesex model with susceptibles classified as having no, partial or full natural resistance to HIV infection, and infecteds classified as rapid, normal or slow progressors. Our goals are to investigate the impact of such heterogeneity on the spread of HIV and to identify key parameters. The basic reproductive number $R_{0}$ is derived from a simplified model. The relative contributions to $R_{0}$ from the three groups of infecteds are investigated. We present a rough estimating procedure making use of limited data to estimate some new parameters specific to our model. Finally the rough estimating procedure is applied to an example focusing on CCR5- $\triangle 32$ in San Francisco gay men. The relative contributions to $R_{0}$ among the three infected groups are compared using two different classifying criteria for infecteds. Under given assumptions, we conclude that, without any intervention, HIV infection will continue to spread in this population and the epidemic is mainly driven by the normal progressors. The transmission rates from infecteds are identified as key parameters.
\end{abstract}

Keywords: HIV, AIDS, mathematical model, $R_{0}$, homosexual, genetics, mutation, CCR5

\section{INTRODUCTION}

The studies of Sheppard, Lang and Ascher (1993) and Phair (1994) find that about $10-15 \%$ of HIV infected individuals remain AIDS free for 10 years or longer (non-progressors), while another $10 \%$ progress within the first 2-3 years (rapid progressors). On the other hand, many individuals remain seronegative even after multiple exposures to HIV from infected partners (Detels et al. 1994, Paxton et al. 1996, Fowke et al. 1996). These distinct outcomes pose an interesting question to HIV/AIDS researchers: What

${ }^{*}$ Corresponding Author: Fax: +41-31-631 38 70; E-mail: sfshsu@math-stat.unibe.ch 
makes the difference? Genetic heterogeneity among individuals may contribute significantly. Several recent studies have demonstrated the protective effects of certain mutant genes on HIV infection or/and AIDS pathogenesis. For instance, Samson et al. (1996) show that a mutant allele, $\Delta 32$, of CCR5 chemokine receptor gene is present at a high frequency of 0.092 in Caucasian populations. The frequencies of homozygotes and heterozygotes for the mutation are about $1 \%$ and $16 \%$, respectively. However, in a cohort of HIV-1 infected Caucasian patients, the heterozygote frequency is $35 \%$ lower than in the general population and no homozygotes with two $\Delta 32$ alleles are found. These observations suggest $\Delta 32$ may provide, at least partial, resistance to HIV-1 infection. Dean et al. (1996) report the same mutant allele with a similar frequency $(\sim$ $10 \%$ ) in the Caucasian population of the United States. Their results indicate that the homozygotes with two $\Delta 32$ alleles may escape from HIV-1 infection and heterozygous infecteds may have a slower progression than other infecteds.

Recently Quillent et al. (1998) characterize another CCR5 gene mutation, m303, which is present among Europeans at an allele frequency of under $1 \%$. Individuals with genotype $\mathrm{m} 303 / \mathrm{m} 303$ or $\Delta 32 / \mathrm{m} 303$ acquire resistance to HIV- 1 infection. Similarly, the m303 heterozygosity may give partial protection against infection and slow down the progression once infected.

In another chemokine, CCR2, Smith et al. (1997) describe a mutation, 64I, which occurs at an allele frequency of 10-15\% among Caucasians and African Americans. Although this mutant gene dose not seem to provide protection against HIV-1 infection, it does indicate a 2-4 years delay of progression among infecteds. Moreover, the effects of CCR5$\triangle 32$ and CCR2-64I on AIDS progression are determined as genetically independent. Among rapid progressors (AIDS onset less than 3 years since infection), about $38-45 \%$ do not have either of the two mutant alleles, $\triangle 32$ and $64 \mathrm{I}$; while among nonprogressors (avoid AIDS for 16 years or more), about $28-29 \%$ can be explained by a mutant allele in either gene.
In stromal-derived factor (SDF-1, the principal ligand for CXCR4), Winkler et al. (1998) also identify a gene variant, 3'A, that shows recessive restriction on AIDS pathogenesis. HIV infected individuals with SDF1-3'A/3'A (homozygous recessive) genotype have a significantly lower relative hazard to AIDS onset and the protection is approximately twice that seen with CCR2 or CCR5 protection. Moreover, CCR and SDF1 protection seem to be additive.

All the information above clearly indicates the existence of genetic heterogeneity with respect to susceptibility to HIV infection and to rate of AIDS progression in general populations. Such kind of heterogeneity has not been studied in the modeling literature. Our special interest in this paper is to investigate, using mathematical models, the impact of such heterogeneity on the spread of HIV and to identify key parameters. To accommodate the genetic heterogeneity on one side and being limited by data availability on the other side, we propose a deterministic one-sex model with susceptibles classified as having no, partial or complete resistance to HIV infection and infecteds as rapid, normal or slow progressors. The details of the general model are presented in Section 2. Under some simplifying assumptions, the basic reproductive number, $R_{0}$ (Diekmann et al. 1990), is obtained in terms of model parameters in Section 3. Based on the limited available information and scarce data, a rough estimation of some parameters is carried out in Section 4. In Section 5 the rough estimating procedure is applied to an example focusing on CCR5- $\triangle 32$ among gay men in San Francisco. The relative contributions to $R_{0}$ among the three infected groups are compared under two different classifying criteria for infecteds. Finally some concluding remarks are provided in Section 6.

\section{MODEL DESCRIPTION}

As the first step in our efforts to incorporate genetic heterogeneity in epidemiological models, we focus on the simplest possible scenario, i.e., a homosexually-active homogeneously-mixing population, to investigate the role of differential 
susceptibility and pathogenesis in HIV infected populations.

Based on the level of natural resistance to HIV, susceptibles are classified into three groups: no resistance $\left(S_{1}\right)$, partial resistance $\left(S_{2}\right)$ and complete resistance $\left(S_{3}\right)$. We assume that $S_{3}$-individuals never become infected. Similarly, based on AIDS pathogenesis, infecteds are classified into three groups: rapid progressor $\left(I_{1}\right)$, normal progressor $\left(I_{2}\right)$ and slow progressor $\left(I_{3}\right)$. AIDS patients are assumed sexually inactive, thus they do not play a role in HIV transmission and are not included in our model. Throughout this paper, the index $i$ refers to group of susceptibles and the index $j$ to group of infecteds.

We assume that recruitment occurs at a constant rate, $\pi$, to replenish the three susceptible groups with respective fractions, $g_{i}\left(i=1,2,3\right.$ and $\sum_{i} g_{i}=$ $1)$, which are related to the frequencies of relevant genotypes. Although genotype frequencies usually change with time due to random fluctuation or/and the disease, the frequencies in the homosexual population do not affect the frequencies among the newcomers, who are progeny of heterosexual populations. However, heterosexual populations are not included in our model and the dynamic of $g_{i}$ is unknown. Hence, we assume, for convenience, that $g_{i}$ are constant. Because frequencies of mutant alleles are relatively small, it is expected that

$$
g_{1}>g_{2}>g_{3}
$$

that is, a large fraction of individuals has no resistance, a small fraction has partial resistance, and an even smaller fraction has complete resistance.

All individuals are subject to the common percapita natural removal rate, $\mu$. The average number of partners per unit time is denoted by $c_{i}$ ( $i=$ $1,2,3)$ for $S_{i}$-individuals and by $d_{j}(j=1,2,3)$ for $I_{j}$-individuals. The per-capita progression rates for $I_{j}$ individuals are denoted by $\gamma_{j}(j=1,2,3)$. Because $1 / \gamma_{j}$ is the average incubation time of $I_{j}$-individuals, it is obvious that

$$
\gamma_{1}>\gamma_{2}>\gamma_{3}
$$

The infectiousness of $I_{j}$ individuals is reflected by the per-partnership transmission rate, $\beta_{j}(j=1,2,3)$. We assume that rapid progressors $\left(I_{1}\right)$ have the highest viral load, thus are most infectious; and that slow progressors $\left(I_{3}\right)$ have the lowest viral load, thus are least infectious. More specifically, we hypothesize the following relation:

$$
\beta_{1} \geq \beta_{2} \geq \beta_{3} .
$$

It has been shown that the viral load and the infectiousness may change dramatically during the incubation period. However, to incorporate this fact we would need to keep track of the "age" of infection for each individual and end up with a complicated model. For the sake of simplicity, here we assume $\beta_{j}$ are constant as in Anderson, Gupta and May (1991) and in McLean and Blower (1993).

During the partnership between an $S_{2}$-individual and an $I_{j}$-individual, the transmission rate $\beta_{j}$ of the infected partner is reduced to $x_{j} \beta_{j}$, with $0<x_{j}<1$ to account for partial resistance to HIV in $S_{2}$-individuals. Newly infected $S_{i}$-individuals $(i=1,2)$ join the three infected groups with respective proportions $f_{i j}$, which satisfy

$$
0 \leq f_{i j} \leq 1 \text { and } \sum_{j=1}^{3} f_{i j}=1 .
$$

We expect the new infecteds who come from $S_{1}$ to generate a larger fraction of rapid progressors $\left(I_{1}\right)$ and a smaller fraction of slow progressors $\left(I_{3}\right)$ than those coming from $S_{2}$, that is,

$$
f_{11}>f_{21} \text { and } f_{13}<f_{23} \text {. }
$$

Because we are looking at a homosexually active population, processes of pair formation and dissolution are not followed explicitly, instead, a proportional mixing pattern is assumed. The total number of sexual partnerships is defined as

$$
\Lambda:=\sum_{i=1}^{3} c_{i} S_{i}+\sum_{j=1}^{3} d_{j} I_{j} .
$$

For a susceptible, given he pairs (i.e., forms a pair with an individual), the chance of pairing with an $I_{j}$-individual is $d_{j} I_{j} / \Lambda$ (Busenberg and CastilloChavez 1991). Thus, the force of infection for $S_{1}$ individuals is

$$
\sigma_{1}:=\sum_{j=1}^{3} \beta_{j} d_{j} I_{j} / \Lambda
$$


and for $S_{2}$-individuals is

$$
\sigma_{2}:=\sum_{j=1}^{3} x_{j} \beta_{j} d_{j} l_{j} / \Lambda
$$

Hence, the rate of new infections in $S_{i}$-individuals $\left(i=1,2\right.$ ), or the incidence from $S_{i}$, is

$$
\delta_{i}:=c_{i} S_{i} \sigma_{i}
$$

These newly infected individuals enter the class $l_{j}$ $(j=1,2,3)$ at the rate

$$
\rho_{j}:=\sum_{i=1}^{2} f_{i j} \delta_{i},
$$

called "birth" rate or the incidence of $I_{j}$. We denote the overall incidence by $\Omega$ as

$$
\Omega:=\sum_{i=1}^{2} \delta_{i}=\sum_{j=1}^{3} \rho_{j},
$$

where equality holds because of condition (4). We are now ready to present our mathematical model:

$$
\begin{aligned}
& \dot{S}_{1}=g_{1} \pi-\mu S_{1}-\delta_{1} \\
& \dot{S}_{2}=g_{2} \pi-\mu S_{2}-\delta_{2} \\
& \dot{S}_{3}=g_{3} \pi-\mu S_{3} \\
& \dot{I}_{1}=\rho_{1}-\left(\mu+\gamma_{1}\right) I_{1} \\
& \dot{I}_{2}=\rho_{2}-\left(\mu+\gamma_{2}\right) I_{2} \\
& \dot{I}_{3}=\rho_{3}-\left(\mu+\gamma_{3}\right) I_{3}
\end{aligned}
$$

In order to make this model analytically tractable, some simplifications are required. A simplified model and the derived basic reproductive number are presented in the next section.

\section{THE BASIC REPRODUCTIVE NUMBER}

Most individuals do not know their genotypes at loci related to HIV susceptibility or/and AIDS pathogenesis, hence, it is reasonable to assume that genetic heterogeneity does not influence pairing behavior. The assumption that all individuals of a given disease status have the same average number of partners per unit time, i.e., $c_{i}=c$ for all $i$ and $d_{j}=d$ for all $j$, is thus not too limiting. To make our model simpler, we further assume that disease status does not affect pairing behavior, i.e., $c=d$, as in Anderson, Gupta and May (1991) and McLean and Blower (1993). In addition, we reparameterize the transmission rates via $\beta:=\beta_{2}$, or more specifically,

$$
\beta_{1}=b_{1} \beta \text { and } \beta_{3}=b_{3} \beta \text {. }
$$

Relation (3) implies that the multipliers

$$
b_{1} \geq 1 \text { and } b_{3} \leq 1
$$

Currently there are no data that throw some light on whether or not the reduction factors $x_{j}$ for $\beta_{j}$ depend on $j$. To continue our goal of analyzing the simplest possible genetic-epidemiological model, we assume that $x_{j}=x$ for all $j$. Relevant variables can now be simplified as follows:

$$
\begin{aligned}
\Lambda & =c\left(\sum_{i=1}^{3} S_{i}+\sum_{j=1}^{3} I_{j}\right) ; \\
\sigma_{1} & =c \beta \sum_{j=1}^{3} b_{j} I_{j} / \Lambda \\
& =\beta \sum_{j=1}^{3} b_{j} I_{j} /\left(\sum_{i=1}^{3} S_{i}+\sum_{j=1}^{3} I_{j}\right) ; \\
\sigma_{2} & =c \beta x \sum_{j=1}^{3} b_{j} I_{j} / \Lambda=x \sigma_{1} ; \\
\delta_{1} & =c S_{1} \sigma_{1} ; \\
\delta_{2} & =c S_{2} \sigma_{2}=x c S_{2} \sigma_{1} ; \\
\rho_{j} & =c \sigma_{1}\left(f_{1 j} S_{1}+x f_{2 j} S_{2}\right), \quad j=1,2,3 .
\end{aligned}
$$

To study the potential of disease spreading, we shall compute the basic reproductive number, $R_{0}$ (Diekmann et al. 1990), which indicates whether a disease may invade a population in demographic steady state when there is no disease present. The computation is done by linearizing our system (12) around the disease-free state and looking for conditions that guarantee the growth of the three infected classes, $I_{j}$. The resulting 3-dimensional system is represented in the following form:

$$
\dot{\mathbf{X}}=(\mathbf{M}-\mathbf{D}) \mathbf{X},
$$


where

$$
\begin{aligned}
& \mathbf{X}=\left[\begin{array}{l}
I_{1} \\
I_{2} \\
I_{3}
\end{array}\right], \quad \mathbf{M}=c \beta\left[\begin{array}{lll}
b_{1} \tau_{1} & \tau_{1} & b_{3} \tau_{1} \\
b_{1} \tau_{2} & \tau_{2} & b_{3} \tau_{2} \\
b_{1} \tau_{3} & \tau_{3} & b_{3} \tau_{3}
\end{array}\right], \\
& \mathbf{D}=\left[\begin{array}{ccc}
\mu+\gamma_{1} & 0 & 0 \\
0 & \mu+\gamma_{2} & 0 \\
0 & 0 & \mu+\gamma_{3}
\end{array}\right],
\end{aligned}
$$

with

$$
\tau_{j}=f_{1 j} g_{1}+x f_{2 j} g_{2}
$$

The three eigenvalues of the matrix $\mathbf{M D}^{-1}$ are 0 , 0 and $\lambda$, where

$$
\begin{aligned}
\lambda & =c \beta\left\{\frac{b_{1} \tau_{1}}{\mu+\gamma_{1}}+\frac{\tau_{2}}{\mu+\gamma_{2}}+\frac{b_{3} \tau_{3}}{\mu+\gamma_{3}}\right\} \\
& =K\left\{Q_{1}+1+Q_{3}\right\}
\end{aligned}
$$

with

$$
\begin{aligned}
K & =c \beta \frac{\tau_{2}}{\mu+\gamma_{2}}, \\
Q_{1} & =b_{1}\left(\frac{\mu+\gamma_{2}}{\mu+\gamma_{1}}\right)\left(\frac{\tau_{1}}{\tau_{2}}\right) \\
\text { and } Q_{3} & =b_{3}\left(\frac{\mu+\gamma_{2}}{\mu+\gamma_{3}}\right)\left(\frac{\tau_{3}}{\tau_{2}}\right) .
\end{aligned}
$$

Because all elements on the right hand side of (24) are positive, it is clear $\lambda>0$. Therefore, $\lambda$ is the dominant eigenvalue of $\mathbf{M D}^{-1}$, which is also referred to the basic reproductive number, $R_{0}$ (Diekmann et al. 1990 ). If $R_{0}>1$, then the disease will successfully invade. Hence, it is important to evaluate the relative contribution of each infected group, determined by $b_{j}$, $\tau_{j}$ and $\gamma_{j}$, to $R_{0}$. If $Q_{1}<1$ and $Q_{3}<1$, then the group of normal progressors, $I_{2}$, contributes the major part, $K$, to $R_{0}$. Under this situation, if $K>1$, then certainly $R_{0}>1$, which implies the disease will spread; if $K<1$, it may still be possible to have $R_{0}>1$ when $K, Q_{1}$ and $Q_{3}$ are not too small. The definitions of $\gamma_{j}$ (2) and $b_{j}$ (14) imply that, in $Q_{1}, b_{1} \geq 1$ and $\left(\mu+\gamma_{2}\right) /\left(\mu+\gamma_{1}\right)<1$. The third term $\tau_{1} / \tau_{2}$ may be larger or smaller than unity, depending on $f_{i j}$. Similarly in $Q_{3}, b_{3} \leq 1$ and $\left(\mu+\gamma_{2}\right) /\left(\mu+\gamma_{3}\right)>1$, while the third term $\tau_{3} / \tau_{2}$ is indeterminant. Overall, it is difficult to compare the magnitudes of $Q_{1}$ and $Q_{3}$ without knowing more precisely the values or ranges of the parameters involved. Further issues about $R_{0}$ are discussed in Section 5, where known values of most parameters are used.

\section{ESTIMATION OF NEW PARAMETERS}

Estimates of most social-demographic and biomedical parameters (e.g., per-capita natural removal rate, $\mu$; recruitment rate, $\pi$; average number of partners per unit time, $c$; overall per-partnership transmission rate. $\beta$; and overall per-capita rate of progression, $\gamma$ ) are readily available in the literature. The additional parameters specific to our model include the distributing fractions $\left(g_{i}\right)$ for newly accrued susceptibles, per-partnership transmission rates $\left(\beta_{j}\right)$ and per-capita rates of progression $\left(\gamma_{j}\right)$ for different infected groups, reduction factor $(x)$ for $\beta_{j}$, and distributing fractions $\left(f_{i j}\right)$ for newly infected individuals. The $g_{i}$ can be estimated from allele or genotype frequencies. The definitions of rapid, normal and slow progressors should give some hints about $\gamma_{j}$. However, there is no direct information about $\beta_{j}, x$ and $f_{i j}$. For the moment we treat $\beta_{j}$ and $x$ as free parameters. To have an educated guess at the values of $f_{i j}$, some "retrospective" information, like frequencies of protective genotypes among infecteds categorized by incubation duration (e.g., Figure 2 in Smith et al. 1997 and Figure 2(B) in Winkler et al. 1998), is used. Let $h_{j i}$ denote the observed fraction of infecteds in $I_{j}$ who were in $S_{i}$; that is, $h_{j i}$ give "retrospective" information on $f_{i j}$. By definition, $\sum_{i=1}^{2} h_{j i}=1$ for $j=1,2,3$. The rough estimates of the fractions of rapid, normal and slow progressors, denoted respectively by $q_{1}, q_{2}$ and $q_{3}$ with $\sum_{j=1}^{3} q_{j}=1$, among all infecteds are also available (e.g., Sheppard, Lang and Ascher 1993, Phair 1994 and Smith et al. 1997). The $h_{j i}$ and $q_{j}$ are usually estimated based on data cumulatively collected over long periods of time in cohort studies. They do not refer to prevalence nor to incidence, but may be viewed as "average" fractions for the given period of time. Using our simplified model together with some additional assumptions, we now show how the "retrospective" $h_{j i}$ can be used to give an estimate of its "prospective" counterpart, $f_{i j}$. 
First we let $\theta:=S_{2} / S_{1}$. Since the majority of susceptibles are in $S_{1}$, it is expected that $0<\theta<1$. From expressions (11), (18) and (19), we obtain the following:

$$
\begin{aligned}
& \delta_{2}=x \theta \delta_{1} \\
& \Omega=(1+x \theta) \delta_{1} .
\end{aligned}
$$

The available $h_{i j}$ and $q_{j}$ are obtained from cohort studies where individuals did not become seropositive all at the same time. Moreover, the longitudinal patterns of $h_{j i}$ and $q_{j}$ are not known. To make rough use of these fractions, we assume that they are constant over time. This assumption helps relate them with the incidence associated with individuals who seroconverted at about the same time. With the given $q_{j}$, the incidence of $I_{j}$ can be expressed as

$$
\rho_{j}=q_{j} \Omega=q_{j}(1+x \theta) \delta_{1}
$$

In our notation and under the above-mentioned assumption, the "retrospective" information about the incidence is described by

$$
\begin{aligned}
& h_{j 1}:=\frac{f_{1 j} \delta_{1}}{\rho_{j}}=\frac{f_{1 j}}{q_{j}(1+x \theta)}, \\
& h_{j 2}:=\frac{f_{2 j} \delta_{2}}{\rho_{j}}=\frac{f_{2 j} x \theta}{q_{j}(1+x \theta)},
\end{aligned}
$$

for $j=1,2,3$. Rearranging the above equations, the $f_{i j}$ can then be expressed as

$$
\begin{aligned}
& f_{1 j}=h_{j_{1} q_{j}}(1+x \theta), \\
& f_{2 j}=h_{j 2} q_{j}(1+x \theta) / x \theta .
\end{aligned}
$$

Summing the above two equations over $j$ and making use of condition (4), one obtains

$$
\begin{aligned}
& 1=(1+x \theta) \eta_{1}, \\
& 1=\left(\frac{1+x \theta}{x \theta}\right) \eta_{2},
\end{aligned}
$$

where

$$
\eta_{1}=\sum_{j} h_{j 1} q_{j} \text { and } \eta_{2}=\sum_{j} h_{j 2} q_{j}
$$

are known quantities. Here the unknown quantity involving $x \theta$ can be evaluated by either

$$
1+x \theta=\frac{1}{\eta_{1}}
$$

or

$$
\frac{1+x \theta}{x \theta}=\frac{1}{\eta_{2}}=\frac{1}{1-\eta_{1}} .
$$

Plugging the results of expressions (37) and (38) respectively into Equations (32) and (33) gives the required estimates for $f_{i j}$.

We are aware of a potential problem with this rough estimating procedure. The quantities $\eta_{1}$ and $\eta_{2}$ come from $q_{j}$ and $h_{j i}$ which are assumed constant over time. This assumption may result in strange behaviors. For instance, from expression (37) one obtains

$$
x \theta=\frac{1}{\eta_{1}}-1=\frac{1-\eta_{1}}{\eta_{1}} .
$$

With constant $x$ and $\eta_{1}$, Equation (39) implies $\theta$ should be constant as well, which is not true in general, but is true when the population is at equilibrium. However, when the population is not at equilibrium, the estimates of $f_{i j}$ may still be reasonable if $\theta$ does not change much over time.

Although the above estimating procedure does not have a sound statistical base and depends on strong assumptions, it may provide sensible guesses, which may be useful especially under the current situation. Furthermore, it is important to note that, in some sense, this is the best that we can hope for given the fact that the available clinical data were not collected to address the questions raised by our model. Lack of data should not constrain the type of questions that may be raised. In fact, we hope that our model results may motivate the collection of the data required here. The application of this rough estimating procedure will be illustrated in the following example.

\section{EXAMPLE}

We choose the population of gay men in San Francisco as target with the focus on the mutation CCR5$\Delta 32$ to illustrate the estimating procedure for $f_{i j}$ as 
well as to assess the relative contributions of the three infected groups to $R_{0}$. Whenever possible, we take parameter values specific for this population; otherwise we take values from other Caucasian AIDS cohorts.

As in McLean and Blower (1993), we use $1 / \mu=32$ years, $\pi=2000 /$ year and $1 / \alpha=5 / 3$ years. As for the value of $\beta c$, because normal progressors are the majority in the infected population, we assume $\beta_{2} c$ can be described by the parameter values estimated for the whole infected population, e.g., $\beta_{2} c=$ $\beta c=0.62 /$ year. No information about $\beta_{1}$ and $\beta_{3}$, or equivalently about $b_{1}$ and $b_{3}$, is available, thus they are considered as free parameters. According to data on homosexually active men in the San Francisco City Clinic Cohort (SFCC) presented in Dean et al. (1996, Table II), the frequencies of the three genotypes are estimated as $g_{1}=0.75, g_{2}=0.23$ and $g_{3}=0.02$. Based on pooled data of Caucasians in five AIDS cohorts (including SFCC) presented in Smith et al. (1997, Figure 3), we define that rapid progressors have an incubation time of less than 3.5 years, slow progressors of more than 13 years, and normal progressors of in between 3.5 and 13 years; i.e., $1 / \gamma_{1}<3.5$ years, 3.5 years $\leq 1 / \gamma_{2} \leq 13$ years and $1 / \gamma_{3}>13$ years. Accordingly we choose $\gamma_{1}=1 / 2$, $\gamma_{2}=1 / 8$ and $\gamma_{3}=1 / 16$ as an educated reasonable guess. When the AIDS criterion of 1993 is applied to these data (top panel in Figure 3 of Smith et al. 1997), the fractions of these three groups are approximated by $q_{1}=0.115, q_{2}=0.645$ and $q_{3}=0.240$, and the within group distributions are approximated by $h_{11}=0.89, h_{12}=0.11, h_{21}=0.81, h_{22}=0.19, h_{31}=$ 0.72 and $h_{32}=0.28$. These values are only approximations due to three reasons: 1) they are estimated from a figure, not directly from counts; 2) this figure is constructed based on pooled data of five AIDS cohorts; and 3) in the figure CCR2-64I mutation is also considered in addition to CCR5- $\Delta 32$ mutation. However, these are the best approximations that we can get from published, aggregated data. We note that the frequency of heterozygotes in two European cohorts of seropositive Caucasians is $35 \%$ lower than in the general populations (Samson et al. 1996). Hence, we select the value of $x=1-0.35=0.65$.
With the above values of $q_{j}$ and $h_{j i}$ the $\eta_{1}$ in (36) is calculated as 0.799 , resulting in $(1+x \theta)=1.254$. Using (32) and (33), we obtain $f_{11}=0.128, f_{12}=$ $0.655, f_{13}=0.217, f_{21}=0.063, f_{22}=0.605$ and $f_{23}=$ 0.332 . These fractions look reasonable and satisfy conditions (4) and (5). Together with other parameters, the magnitude of $R_{0}$ and relative contributions from the three infected groups can be assessed using (25). Because the value of $x$ is suggested from geographically distinct populations, we decide to treat $x$ first as a free parameter and then take the value of 0.65 for further investigation.

As shown in Figure 1, the relative contribution of rapid progressors, $Q_{1}$, is rather small, ranging between 0.05 and 0.23 with $b_{1} \in[1,4]$ and $x \in(0,1)$, and is more sensitive to $b_{1}$ than to $x$. The upper bound of $b_{1}$ is chosen for illustrative purpose. With $b_{3} \in(0,1]$ and $x \in(0,1)$, the relative contribution of slow progressors, $Q_{3}$, is mostly larger than $Q_{1}$, but still less than unity with a range between 0 and 0.63 . As can be seen from Figure 2, the value of $Q_{3}$ is also more sensitive to $b_{3}$ than to $x$. Because $Q_{1}<1$ and $Q_{3}<1$, the normal progressors contribute the most to $R_{0}$, with $K$ evaluated by

$$
K=1.949+0.552 x \text {. }
$$

For $0<x<1$, we obtain $1.95<K<2.50$, which "guarantees" the disease will continue to spread in this population.

When $x$ is fixed at 0.65 , the magnitude of $R_{0}$ for different values of $b_{1}$ and $b_{3}$ can be calculated using (25) as follows:

$$
R_{0}=2.308\left(0.053 b_{1}+1+0.609 b_{3}\right),
$$

which is also plotted in Figure 3. Because the coefficient of $b_{3}$ is about 11.5 times of that of $b_{1}, R_{0}$ is much more sensitive to value of $b_{3}$ than to $b_{1}$. With $b_{1} \in$ $[1,4]$ and $b_{3} \in(0,1]$, we obtain $0.053 \leq Q_{1} \leq 0.212$ and $0<Q_{3} \leq 0.609$, which are clearly less than unity. Hence, the normal progressors gives the major contribution to $R_{0}$ with $K=2.308>1$ (this fact is generally true for $x \in(0,1)$ as presented in (40)). The resulting range of $R_{0}$ is between 2.43 and 4.20 . When $b_{1}=1$ and $b_{3}=1$, we obtain $R_{0}=3.84$ with $Q_{1}=0.053<Q_{3}=0.609$; while when $b_{1}=4$ and 


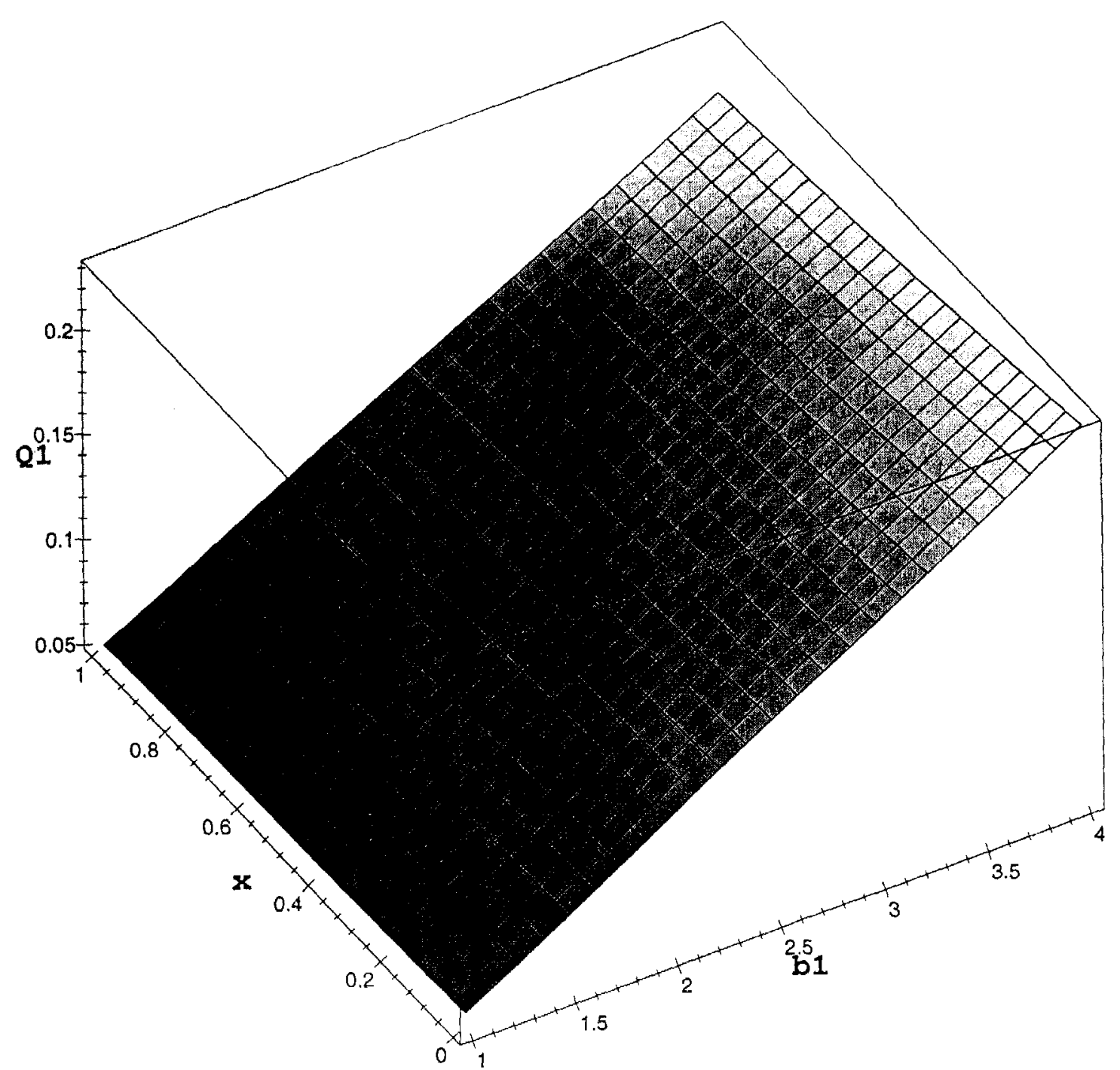

FIGURE 1 Relative contribution to basic reproductive number, $R_{0}$, from the rapid progressors, $Q_{1}$, as a function of the corresponding multiplier of reference transmission rate, $b_{1}$, and the reduction factor for the per-partnership transmission rate, $x$.

$b_{3}=0.25, R_{0}=3.15$ with $Q_{1}=0.212>Q_{3}=0.152$. Thus, depending on the values of $b_{1}$ and $b_{3}$, the relative contribution of rapid progressors may be smaller or larger than that of slow progressors.

The way we classify rapid, normal and slow progressors based on incubation period in the above example is somehow arbitrary. However, there does not seem to be a standard classification presented in the literature. To investigate the effects of the classification, we now repeat the same evaluation with a different cut-off value, $\geq 16$ years, for slow progressors. More specifically, we define rapid progressors as before, but slow progressors have an incubation period of at least 16 years, and thus normal progressors lie between 3.5 and 16 years. The new values for the rates of progression are chosen as $\gamma_{2}=1 / 10$ and $\gamma_{3}=1 / 18$. The relevant fractions approximated from Smith et al. (1997, Figure 3) are $q_{2}=0.845, q_{3}=0.040, h_{21}=0.79, h_{22}=0.21$, $h_{31}=0.77$ and $h_{32}=0.23$. The prospective fractions are calculated as $f_{11}=0.128, f_{12}=0.834, f_{13}=0.038$, $f_{21}=0.063, f_{22}=0.890$ and $f_{23}=0.047$, which again 


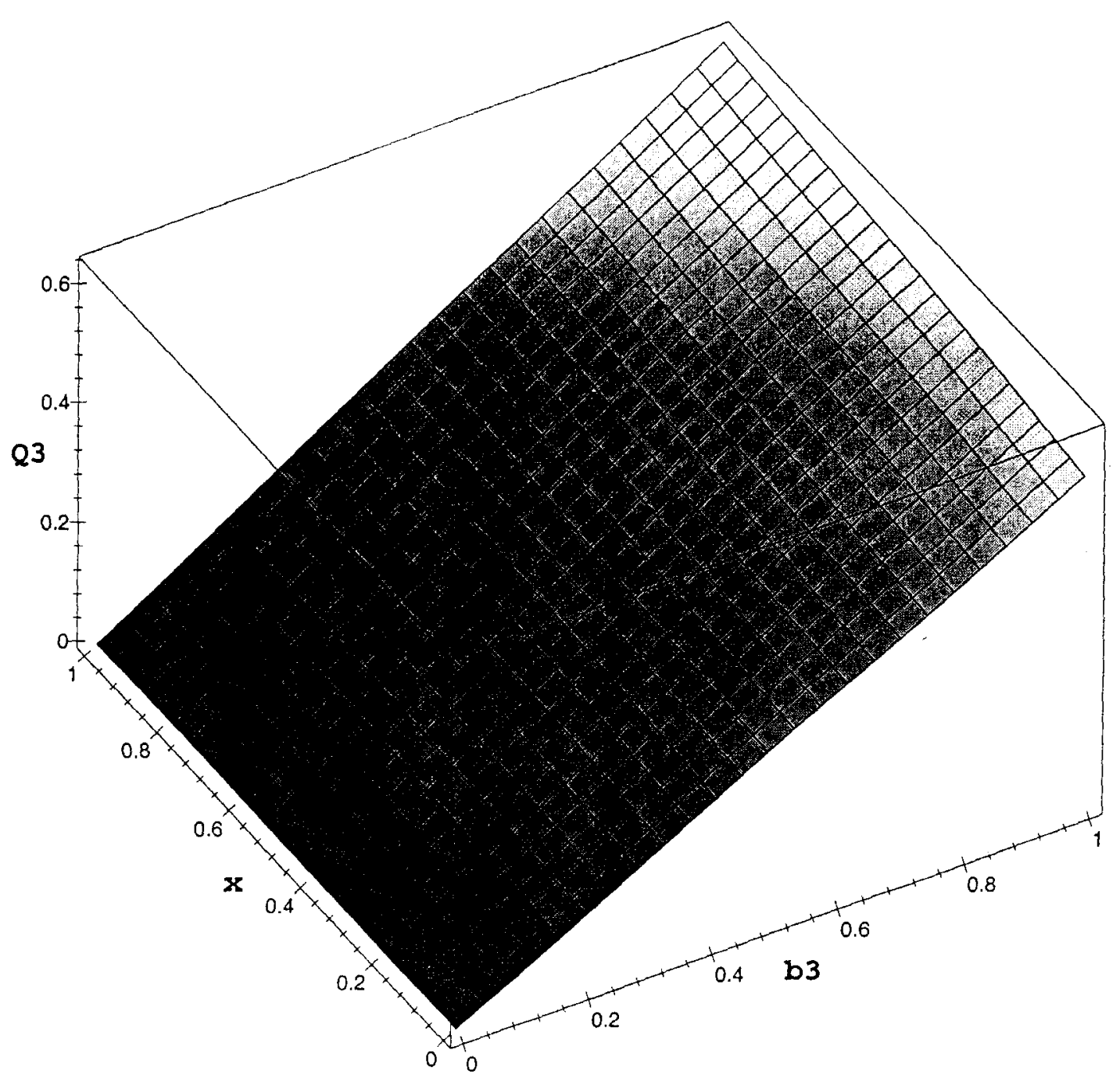

FIGURE 2 Relative contribution to basic reproductive number, $R_{0}$, from the slow progressors, $Q_{3}$, as a function of the corresponding multiplier of reference transmission rate, $b_{3}$, and the reduction factor for the per-partnership transmission rate, $x$.

satisfy conditions (4) and (5). When $x$ is fixed at 0.65 , the magnitude of $R_{0}$ for different values of $b_{1}$ and $b_{3}$ can be calculated by the following formula:

$$
R_{0}=3.583\left(0.034 b_{1}+1+0.071 b_{3}\right)
$$

which is also more sensitive to $b_{3}$ than to $b_{1}$; however, the coefficient of $b_{3}$ is only about 2 times of that of $b_{1}$. With $b_{1} \in[1,4]$ and $b_{3} \in(0,1]$, we obtain $0.034 \leq$ $Q_{1} \leq 0.136$ and $0<Q_{3} \leq 0.071$, which are clearly less than unity. Moreover, $Q_{1}$ and $Q_{3}$ here are smaller than the earlier evaluation due to larger $f_{12}$ and $f_{22}$ in $\tau_{2}$ and a smaller $\gamma_{2}$. For $Q_{3}$ the additional influence comes from much smaller $f_{13}$ and $f_{23}$ in $\tau_{3}$ and a smaller $\gamma_{3}$. The contribution to $R_{0}$ from normal progressors is $K=3.583$, which is larger than the $K=2.308$ in the earlier evaluation also due to larger $f_{12}$ and $f_{22}$ in $\tau_{2}$ and a smaller $\gamma_{2}$. The value of $R_{0}$ ranges between 3.70 and 4.32 , which is also larger than the earlier evaluation. When $b_{1}=1$ and $b_{3}=1$, we obtain $R_{0}=3.96$ with $Q_{1}=0.034<$ $Q_{3}=0.071$; while when $b_{1}=4$ and $b_{3}=0.25, R_{0}=$ 4.14 with $Q_{1}=0.137>Q_{3}=0.018$. As before, the relative magnitude between $Q_{1}$ and $Q_{3}$ depends on the values of $b_{1}$ and $b_{3}$. 


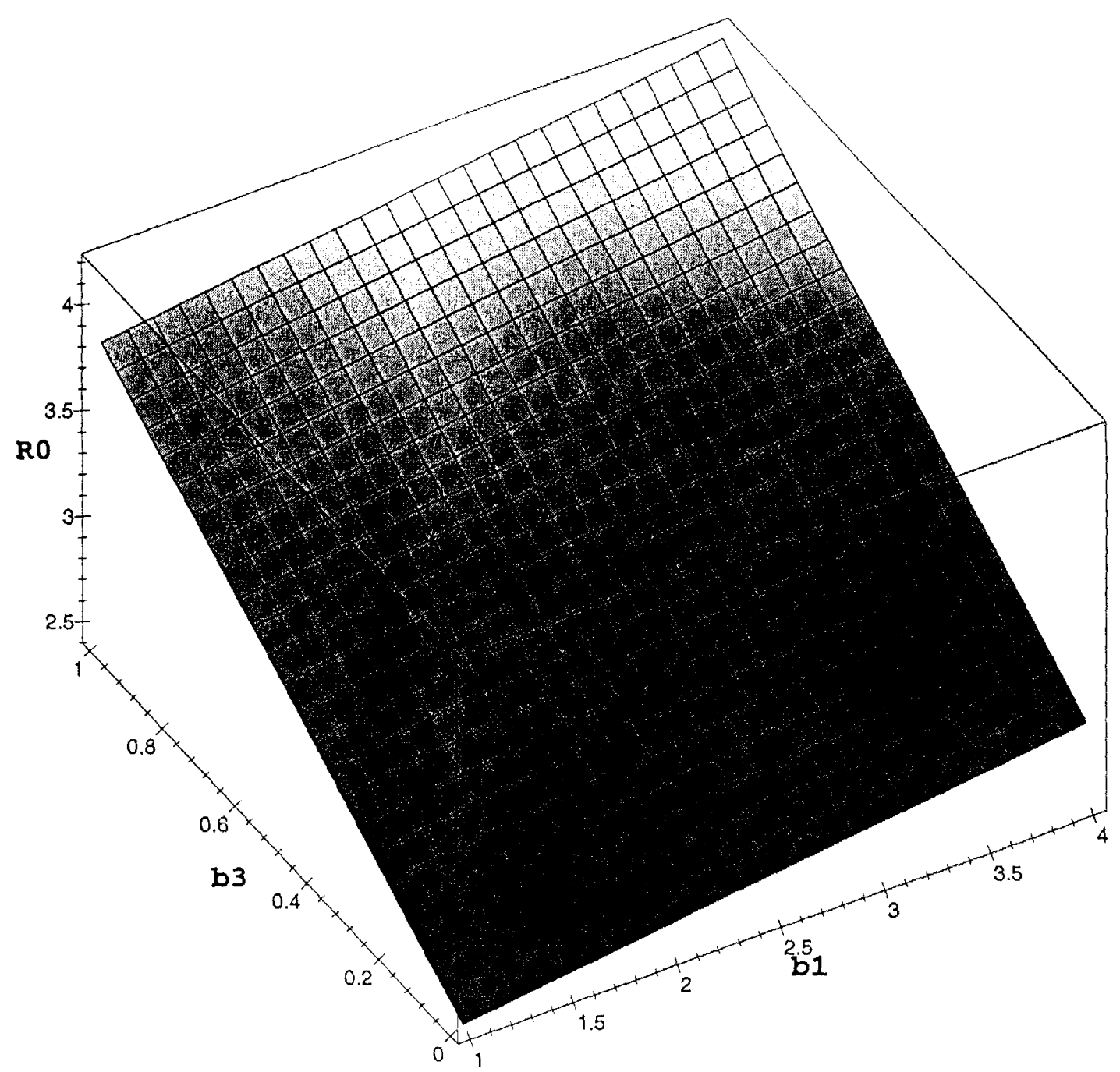

FIGURE 3 Basic reproductive number, $R_{0}$, as a function of the multipliers of reference transmission rate, $b_{1}$ and $b_{3}$, with the reduction factor for the per-partnetship transmission rate, $x=0.65$.

\section{CONCLUDING REMARKS}

We have presented a novel model to incorporate genetic heterogeneity into HIV / AIDS epidemiology. The basic reproductive number for this model has been derived and the relative contributions from different infected groups have been discussed. Because published data are limited, values of some parameters are not available and have to be estimated in a rough way. Our rough estimating procedure for distributing fractions of infecteds, $f_{i j}$, has provided reasonable estimates in the above example.
To improve the accuracy of this estimation, data collected in a prospective manner, e.g., keeping track of how many $S_{i}$ individuals become $I_{j}$ individuals, would be very helpful. However, it may take a long time and a lot of efforts to observe a sufficient number of new infection cases and follow them until AIDS onset. The alternative is to develop more sophisticated estimating procedures to make use of the retrospective data.

Among the three free parameters, i.e., $x, b_{1}$ and $b_{3}$, the basic reproductive number $R_{0}$ for the homosexual population in San Francisco seems to be more 
sensitive to factors of transmission rates, $b_{1}$ and $b_{3}$, than to the reduction factor, $x$. Therefore, more efforts should be devoted to the estimation of $b_{j}$, or equivalently the transmission rates $\beta_{j}$. When $x=0.65$, the value of $R_{0}$ lies between 2.43 and 4.32 , depending on classifying criterion for infecteds and values of $b_{1}$ and $b_{3}$. Anyway, it clearly indicates that HIV infection will continue to spread in this population and the major contribution to $R_{0}$ is from the normal progressors, who are the majority among infecteds. This implies HIV prevention and treatment interventions should certainly include this major group. Further investigation on effects of treatment and vaccination in this population will be published elsewhere (Hsu Schmitz 1999). The relative contribution from rapid progressors may be smaller or larger than that from slow progressors, again depending on classifying criterion for infecteds and values of $b_{1}$ and $b_{3}$. A standard classifying criterion for infecteds will be welcomed and is awaited, so we will have less confusion when several studies are compared.

The estimation and evaluation applied to the example are mainly for illustrative purpose. The assumptions required by the model and the rough estimating procedure might not all be satisfied in the example population. For instance, the fractions $q_{j}$ and $h_{j i}$ might not be constant. Furthermore, treatment is commonly used in this population in recent years. Our model without considering treatment effects might not reflect the real epidemic. However, the main purposes of this exercise are: 1) to motivate clinical researchers to collect required data (e.g., $\gamma_{j}, \beta_{j}, x$ and $f_{i j}$ ) and to standardize the classifying criterion for infecteds; and 2) to identify key parameters, e.g., $b_{1}$ and $b_{3}$.

In our model infecteds coming from $S_{1}$ and those from $S_{2}$ are pooled together. It would be more informative, and probably also more realistic, to have three infected groups specifically for $S_{1}$-individuals and another three infected groups for $S_{2}$-individuals. However, this will give three equations more in the model and make the analysis and interpretation more difficult. Moreover, this requires additional parameters to be estimated from more detailed data, which are not readily available.
As mentioned in the Introduction, there are several mutant alleles of different loci related to susceptibility to HIV or/and rate of progression to AIDS. Thus, it may be more appropriate to consider a combined locus accommodating several relevant loci, instead of focusing on a single locus. For instance, Smith et al. (1997) combine the CCR5 locus and the CCR2 locus into a compound locus. More data should also be collected to cover this aspect.

\section{Acknowledgement}

The valuable comments from two reviewers and Carlos Castillo-Chavez have improved this paper to a large extent. This research was supported by the Swiss NSF grant No. 20-49319.96.

\section{References}

[1] Anderson, R. M., Gupta, S. and May, R. M. (1991). Potential of community-wide chemotherapy or immunotherapy to control the spread of HIV-1. Nature, 350, 356-359.

[2] Busenberg, S. and Castillo-Chavez, C. A. (1991). A general solution of the problem of mixing of subpopulations and its application to risk- and age-structured epidemic models for the spread of AIDS. IMA Joumal of Mathematics Applied in Medicine and Biology, 8, 1-29.

[3] Dean, M., Carrington, M., Winkler, C., et al. (1996). Genetic restriction of HIV -1 infection and progression to AIDS by a deletion allele of the CKR5 structural gene. Science, 273, $1856-1862$.

[4] Detels, R., Liu, Z., Hennessey, K., et al. (1994). Resistance to HIV-1 infection. Multicenter AIDS Cohort Study. J Acquir Immune Defic Syndr Hum Retrovirol, 7, 1263-1269.

[5] Diekmann, O., Heesterbeek, J. A. P. and Metz, J. A. I. (1990). On the definition and the computation of the basic reproduction ration $R_{0}$ in models for infectious diseases in heterogeneous populations. Journal of Mathematical Biology, 28, 365-382.

[6] Fowke, K. R., Nagelkerke, N. J. D., Kimani, J., et al. (1996). Resistance to HIV-1 infection among persistently seronegative prostitutes in Nairobi, Kenya. Lancet, 348, 1347-1351.

17] Hsu Schmitz, S.-F. Treatment and vaccination against HIV/ AIDS in homosexuals with genetic heterogeneity. Mathematical Biosciences, (to appear)

[8] McLean, A. R. and Blower, S. M. (1993). Imperfect vaccines and herd immunity to HIV. Proc. R. Soc. Lond. B, 253, $9-13$.

[9] Paxton, W. A., Martin, S. R., Tse, D., et al. (1996). Relative resistance to HIV-1 infection of CD4 lymphocytes from persons who remain uninfected despite multiple highrisk sexual exposure. Nature Medicine, 2, 412-417.

[10] Phair, J. P. (1994). Keynote address: variations in the natural history of HIV infection. AIDS REs Hum REtrovir, 10, $883-885$.

[11] Quillent, C., Oberlin, E., Braun, J, et al. (1998). HIV-1resistance phenotype conferred by combination of two separate inherited mutations of CCR5 gene. The Lancet, 351, $14-18$ 
[12] Samson, M., Libert, F., Doranz, B. J., et al. (1996). Resistance to HIV-1 infection in caucasian individuals bearing mutant alleles of the CCR-5 chemokine receptor gene. Nature, 382, 722-725.

[13] Sheppard, H. W., Lang, W. and Ascher, M. S. (1993). The characterization of non-progressors: long-term HIV-I infection with stable CD4+ T-cell levels. AIDS, 7, 1159-1166.
[14] Smith, M. W., Dean, M., Carrington, M., et al. (1997). Contrasting genetic influence of CCR2 and CCR5 variants on HIV-1 infection and disease progression. Science, 277, 959-965.

[15] Winkler, C., Modi, W., Smith, M. W., et al. (1998). Genetic restriction of AIDS pathogenesis by an SDF-1 chemokine gene variant. Science, 279, 389-393. 


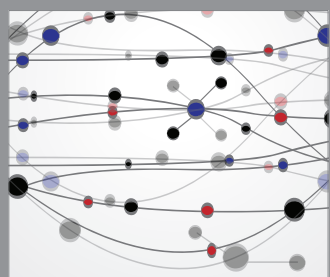

The Scientific World Journal
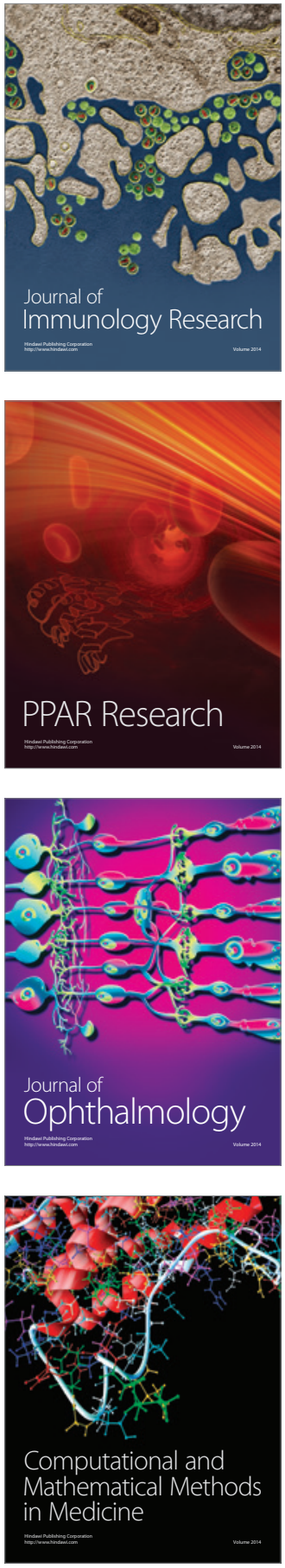

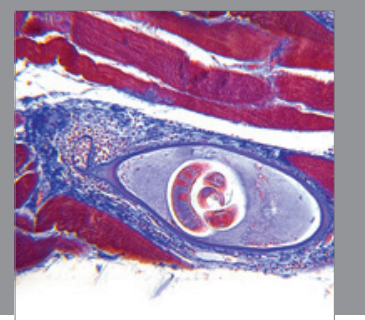

Gastroenterology

Research and Practice
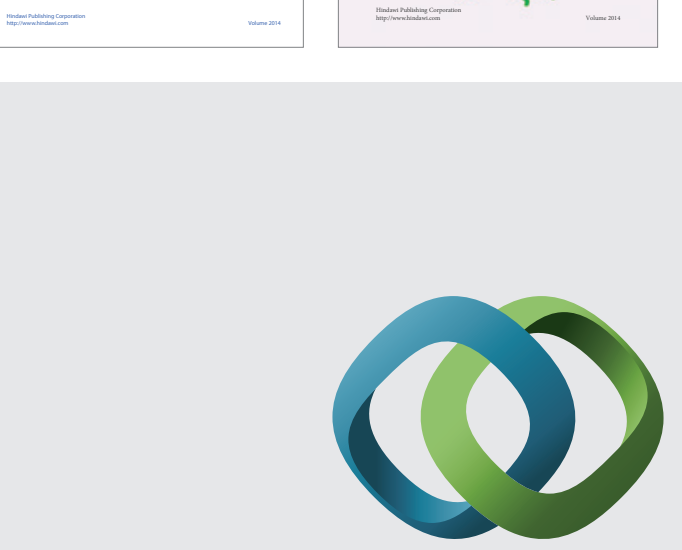

\section{Hindawi}

Submit your manuscripts at

http://www.hindawi.com
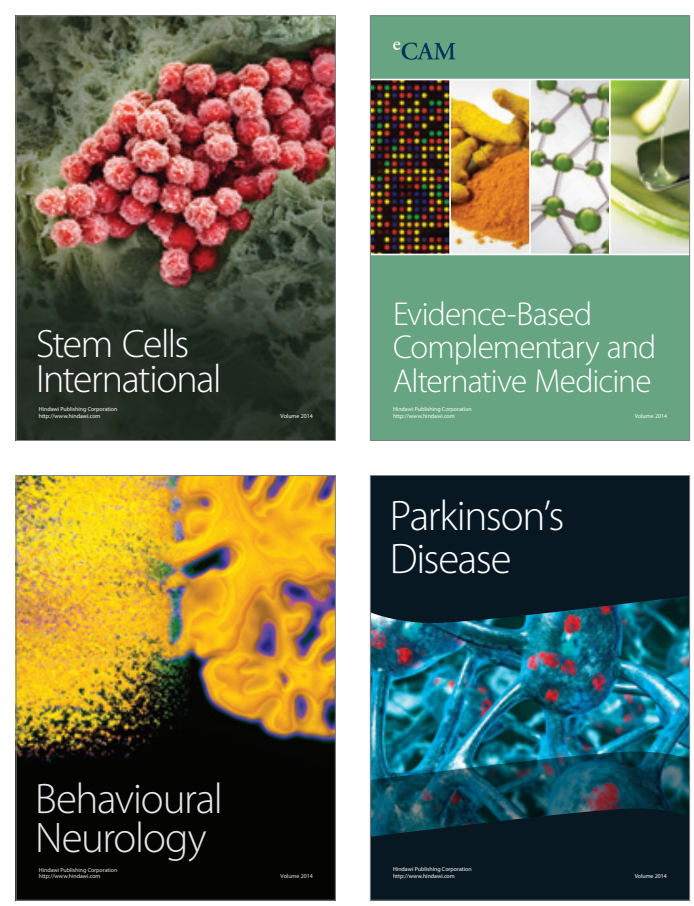

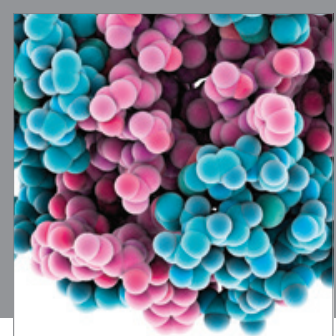

Journal of
Diabetes Research

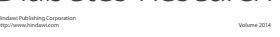

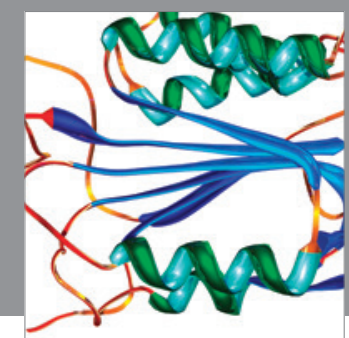

Disease Markers
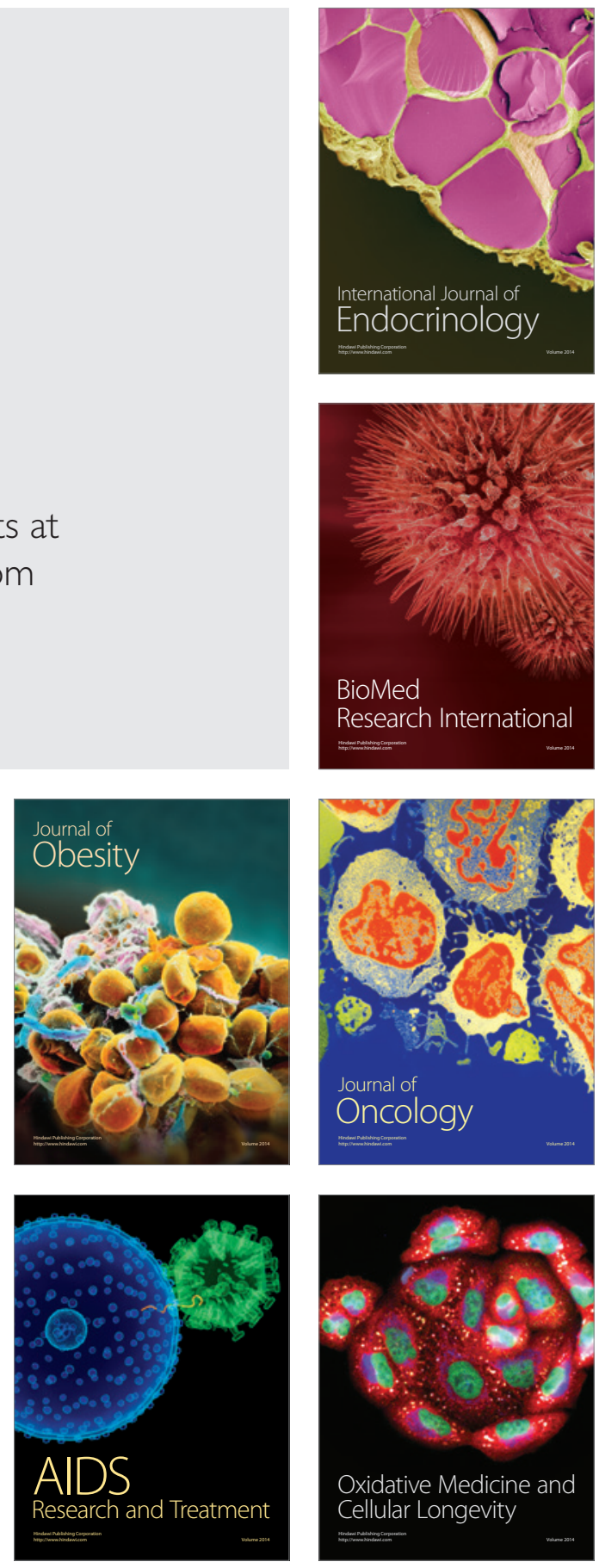